\section{ORIGINAL RESEARCH}

C.M. Strother

F. Bender

Y. Deuerling-Zheng

K. Royalty

K.A. Pulfer

J. Baumgart

M. Zellerhoff

B. Aagaard-Kienitz

D.B. Niemann

M.L. Lindstrom

\title{
Parametric Color Coding of Digital Subtraction Angiography
}

BACKGROUND AND PURPOSE: Color has been shown to facilitate both visual search and recognition tasks. It was our purpose to examine the impact of a color-coding algorithm on the interpretation of 2D-DSA acquisitions by experienced and inexperienced observers.

MATERIALS AND METHODS: Twenty-six 2D-DSA acquisitions obtained as part of routine clinical care from subjects with a variety of cerebrovascular disease processes were selected from an internal data base so as to include a variety of disease states (aneurysms, AVMs, fistulas, stenosis, occlusions, dissections, and tumors). Three experienced and 3 less experienced observers were each shown the acquisitions on a prerelease version of a commercially available double-monitor workstation (XWP, Siemens Healthcare). Acquisitions were presented first as a subtracted image series and then as a single composite color-coded image of the entire acquisition. Observers were then asked a series of questions designed to assess the value of the color-coded images for the following purposes: 1) to enhance their ability to make a diagnosis, 2) to have confidence in their diagnosis, 3) to plan a treatment, and 4) to judge the effect of a treatment. The results were analyzed by using 1-sample Wilcoxon tests.

RESULTS: Color-coded images enhanced the ease of evaluating treatment success in $>40 \%$ of cases $(P<.0001)$. They also had a statistically significant impact on treatment planning, making planning easier in $>20 \%$ of the cases $(P=.0069)$. In $>20 \%$ of the examples, color-coding made diagnosis and treatment planning easier for all readers $(P<.0001)$. Color-coding also increased the confidence of diagnosis compared with the use of DSA alone $(P=.056)$. The impact of this was greater for the naïve readers than for the expert readers.

CONCLUSIONS: At no additional cost in $\mathrm{x}$-ray dose or contrast medium, color-coding of DSA enhanced the conspicuity of findings on DSA images. It was particularly useful in situations in which there was a complex flow pattern and in evaluation of pre- and posttreatment acquisitions. Its full potential remains to be defined.

ABBREVIATIONS: $\mathrm{AP}=$ anteroposterior; $\mathrm{AVF}=$ arteriovenous fistula; $\mathrm{AVM}=$ arteriovenous malformation; DSA = digital subtraction angiography; HSV = hue, saturation, value; $I_{\text {mask }}=$ pixel intensity in the mask frame; $I_{\max }=$ maximal enhancement; $I_{\text {peak }}=$ peak pixel intensity; TTP = time to peak

$\mathbf{T}$ here is good evidence for the use of color in tasks that require both visual search (identification) and visual recognition (searching tasks require that an observer detect a previously defined object or objects; recognition tasks require not only detection but also categorization of an unknown object or objects). ${ }^{1}$ Advantages offered by color-coding of a display increase as the search and recognition tasks become more complex. $^{1,2}$

Early in the development of DSA, there were multiple descriptions of techniques for the extraction and display of temporal information that could then be used to create parametric images of the patterns of blood flow through the vasculature. $^{3,4}$ Such parametric images provided a means to demonstrate functional aspects of the circulation that could not be grasped from viewing either standard DSA image series or single DSA images. ${ }^{3,4}$ The potential value of the use of color in

Received July 16, 2009; accepted after revision September 24

From the University of Wisconsin Hospitals and Clinics (C.M.S., K.A.P., B.A.-K., D.B.N.), Madison, Wisconsin; Siemens AG (F.B., Y.D.-Z., M.Z.), Healthcare Sector, Forchheim, Germany; Siemens Medical Solutions (K.R., J.B.), Angiography Division, Hoffman Estates, Illinois; and Department of Biostatistics (M.L.L.), University of Wisconsin, Madison, Wisconsin

Please address correspondence to Charles Strother, MD, Department of Radiology, University of Wisconsin, 600 N. Highland Ave, Madison, WI 53792; e-mail: strother@wisc.edu DOI 10.3174/ajnr.A2020 the display of these parametric images was recognized but was not, to our knowledge, ever exploited into widespread clinical use. ${ }^{3-5}$ Recently, an algorithm for creation of a composite color-coded time-of-arrival image from a DSA sequence has been described. ${ }^{6}$

Today, DSA is primarily used either for evaluation of complex vascular abnormalities that cannot be adequately assessed by using CT angiography or MR angiography or for monitoring and guidance of minimally invasive endovascular interventions. It was our hypothesis that application of a temporal and intensity color-coded algorithm to DSA acquisitions of a variety of central nervous system vascular lesions would add value to the ability both to diagnose and determine the effects of therapeutic interventions. This report describes our observations in the use of this algorithm.

\section{Materials and Methods}

Under an institutional review board-approved protocol, 26 DSA acquisitions from patients with a variety of central nervous system vascular abnormalities were selected from an internal data base for review. Image series were chosen from subjects who had undergone angiography during the period between January 1, 2007, and September 1, 2008, either for diagnosis or treatment of aneurysms, AVMs/ fistulas, vasospasm, stenosis/occlusions/dissections, or tumors. 
Table 1: Categories of disease states and their numbers included in the study

\begin{tabular}{lc}
\hline Disease & No. of Cases \\
\hline Aneurysm & 5 \\
AVM/fistula & 5 \\
Dissection & 3 \\
Stroke/embolism & 2 \\
Stenosis & 5 \\
Tumor & 2 \\
Vasospasm & 4 \\
\hline
\end{tabular}

Twenty-three cases with pre- and posttreatment studies were included; only cases having similar projections and catheter positions in the pre- and posttreatment studies qualified for inclusion in the study. The category of studies selected along with the number of studies in each category is shown in Table 1 . All pre- and posttreatment angiograms were obtained by using the same catheter, and all were made by using hand injections, for the most part by the same operator. The position of the catheter between the 2 acquisitions varied somewhat. Previous work has shown that within wide variation, the position of a catheter has very limited impact on the geometry of an arterial contrast medium bolus. ${ }^{7}$ Image sequences that were suboptimal because of patient movement were excluded as were ones in which the series did not extend from a time when there was no arterial opacification through a late venous phase of the circulation.

Six readers were selected to include 3 experienced observers and 3 observers with limited experience in cerebral angiography. Two of the experienced observers were staff neurointerventionalists; the other was a senior staff diagnostic neuroradiologist. One of the inexperienced observers was a neurointerventional fellow, one was a diagnostic neuroradiology fellow who had almost 1 year's experience rotating on the angiography/interventional service, and 1 was a senior neurosurgical resident who had almost 1 year's experience performing diagnostic and interventional angiographic procedures. Before looking at the images, each observer was given a short tutorial explaining the basic color-flow algorithm and its application. Each observer viewed the image set separately. Images were viewed on a prerelease version of a commercial workstation (XWP; Siemens Healthcare, Forchheim, Germany). Observers were allowed to manipulate both the standard DSA images (review, window, stop, magnify, etc) and the color-coded images as they would do in clinical practice. Images were divided into 2 groups: 1) those with only pretreatment images, and 2) those with both pre- and posttreatment images. Most of the acquisitions were obtained at the same frame rate. The viewing window was adjusted without regard to frame rate so that by default, the start of the window was the first frame before contrast and the end of the window was the last frame of the run.

Observers were first shown the image set consisting of only pretreatment images according to the following scheme: First, the standard DSA sequence was presented, and the observer was asked to record his or her diagnosis along with the degree of confidence in the diagnosis on a scale of $0 \%-100 \%$. Second, the color-coded image was presented in addition, and the observer was asked, if necessary, to modify his or her diagnosis and also to rate the level of confidence in the diagnosis on the basis of the combination of the standard DSA images and the color-coded image on a scale of $0 \%-100 \%$. Next, observers were asked to answer the following questions by using a response of "easier," "same," or "harder": "What was the impact of the color-coded image on your diagnosis?" and "What was the impact of the color-coded image in terms of treatment planning?"
For the sets with both pre- and posttreatment images, observers were first shown the pre- and posttreatment DSA acquisitions and were then asked to rate the success of treatment by using a 0 (not successful at all) to 100 (complete success) scale. Next they were shown the pre- and posttreatment color-coded images along with the pre- and posttreatment DSA acquisitions and were asked again to rate the level of success of treatment (by using the same scale) on the basis of the availability of both types of images. For this study, treatment success was defined as being a technical end point-that is, did the treatment of vasospasm improve blood flow to the brain or did embolization of an AVM or AVF result in obliteration of some significant component of the abnormality? Finally, they were asked to answer the following questions by using a response of "easier," "same," or "harder": "What was the impact of the color-coded image? Did it make it easier, the same, or harder?"

\section{Image Postprocessing for Application of Color Coding}

The DSA acquisitions were postprocessed by using prototype software. Time-concentration curves were obtained for each individual pixel or cluster of pixels. We extracted 2 kinds of parameters: 1) maximal enhancement $\left(I_{\max }=I_{\text {mask }}-I_{\text {peak }}\right)$, and 2) temporal parameters such as TTP. The $I_{\max }$ was calculated as the difference between the $I_{\text {peak }}$ and $I_{\text {mask }}$, which was predefined as the frame in which contrast agent had not arrived in the structures. The $\mathrm{I}_{\max }$ was then normalized against the maximal $I_{\max }$ among all the pixels in the image, so that all the $\mathrm{I}_{\max }$ values lay in the range of $0-1$.

The TTP and the $\mathrm{I}_{\max }$ were color-coded by using the HSV color space as follows: Hue $=$ TTP $\times 240 / 360$, Saturation $=$ constant, Value $=\mathrm{I}_{\max }$.

With this mapping, the color of a pixel in the resulting composite image can be determined by the TTP and the brightness by the $\mathrm{I}_{\max }$. The saturation was kept as a constant that can be mapped with further parameters. The HSV value of each pixel was then converted to the Red, Green, Blue color presentation by using widely accepted formulas. The processed images derived from DSA acquisitions before and after intervention were displayed in a side-by-side view for comparison.

\section{Statistical Methods}

One-sample (or paired) Wilcoxon tests were used to compare all responses for which the pairing was based on case. Readers' responses were averaged before comparison either across all 6 readers or separately for naïve and experienced readers. Responses with the coding "easier," "same," or "harder" were transformed to numeric scores as easier $=1$, same $=0$, and harder $=-1$. To assess inter-rater variability, we used intraclass correlations by calculating the maximum number of readers who agreed for each case, then computing the mean and standard error of that number over all the cases (because there were 6 readers, a mean of 6 would mean perfect agreement).

\section{Results}

In $>40 \%$ of the cases $(P<.0001)$, both experienced and naïve observers found that the color-coded images enhanced their ability to determine whether a therapeutic intervention had been successful. This benefit was most helpful in those cases in which either postsubarachnoid vasospasm or an intracranial stenosis had been treated. The composite color-coded image much more clearly depicted improvements both in the speed and extent of parenchymal opacification (Figs 1 and 2). In subjects with vasospasm or stenosis, the color-coded images of 

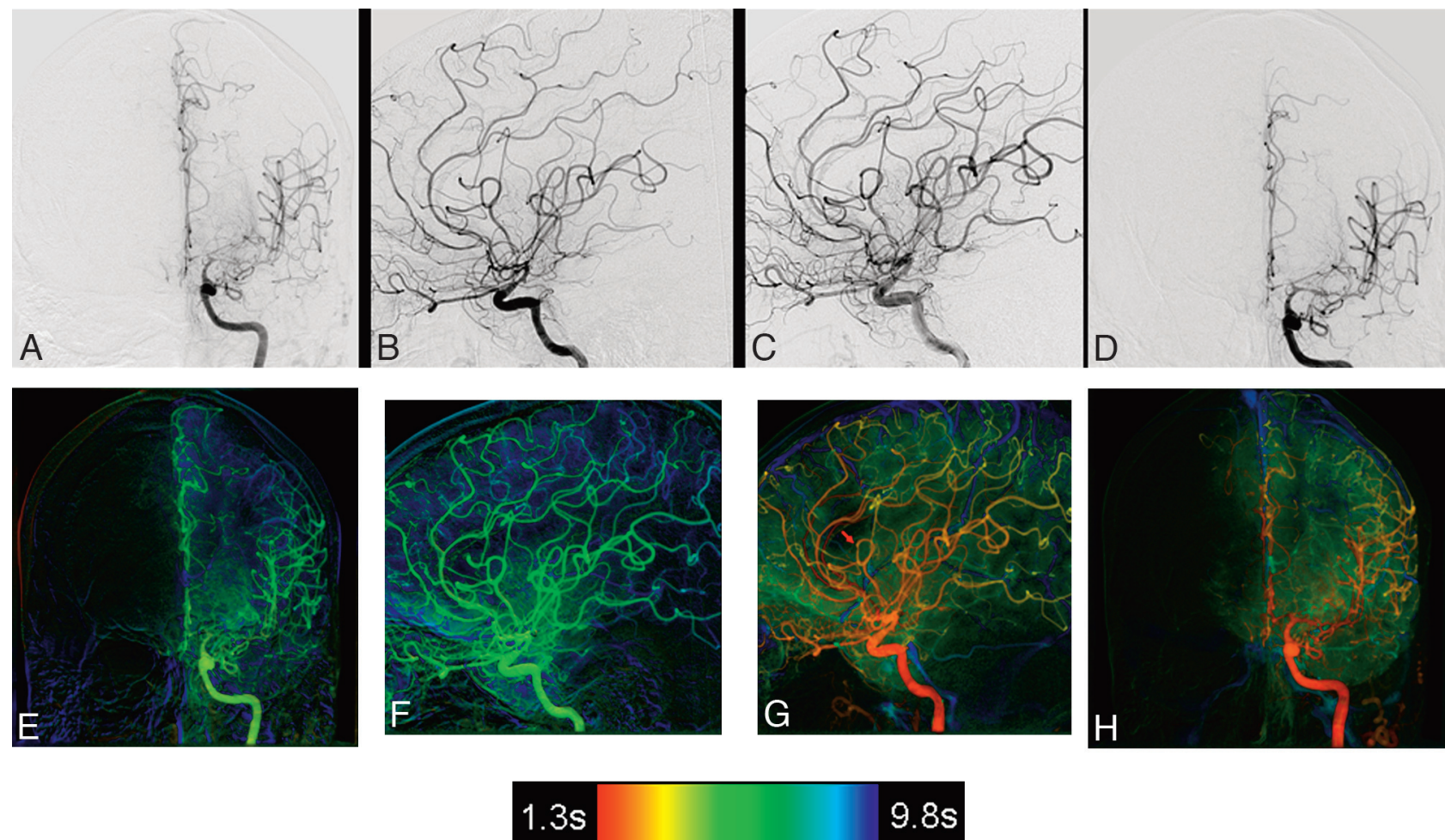

Fig 1. $A-D$, Top row: $A P$ and lateral left carotid angiograms pre- $(A$ and $B)$ and postinfusion $(C$ and $D)$ and angioplasty for treatment of subarachnoid hemorrhage vasospasm. Bottom row: Corresponding AP and lateral color-coded images of the DSA acquisitions shown in the top row. $E-H$, Pretreatment $(E$ and $A$ ) and posttreatment $(G$ and $H$ ). In addition to improvements in the caliber of the proximal arteries easily seen on the standard DSA images, the color-coded composites show faster and more complete contrast filling of the parenchyma. A red arrow $(G)$ shows that a small middle cerebral artery has been occluded during the treatment. A wedge of no filling in this arterial distribution is easily seen on the color-coded image.

DSA sequences obtained before treatment made it easier for observers to plan a therapeutic intervention in that they allowed clearer visualization of slow or incomplete parenchymal opacification in tissue downstream from a stenosis, blockage, or vasospasm than did the DSA sequence (Figs 1 and 2). In subjects with an AVM/AVF, the ability to visualize arterial feeders, abnormal arterialized veins, and normal veins on a composite image was thought to add value to both endovascular and open surgical treatment planning (Fig 3). In these cases, the readers found that the composite color-coded image made it easier for them to understand both the anatomy and the flow characteristics of the lesions. In $>20 \%$ of these cases, this extra information was thought to add significant value to their treatment planning $(P=.0069)$.

The benefit of the color-coded images extended to making both diagnosis and treatment planning easier for all readers in $>20 \%$ of the cases $(P<.0001)$, while also improving the observers' confidence in their diagnoses compared with using DSA alone $(P=.056)$. While this impact was greater for the naïve readers than for the experienced observers, this difference was not found to be statistically significant. As with many types of studies, there was agreement among the readers that the color-coded images helped them; however, there were some disagreements as to just how this value was added (Table 2). Some qualitative comments from the readers illustrate this point. In relation to 1 of the AVMs, an experienced observer commented, "It was easier to see slow or fast flow in veins on the color image than on the DSA images." In relation to 1 of the vasospasm cases, a less-experienced observer commented, "The color-flow images showed improvement in flow better than the angio." An experienced observer commented about another vasospasm case that "the very slow transit time was striking on the color-flow image."

\section{Discussion}

We have demonstrated some of the potential benefits derived from application of a temporal and intensity color-coded algorithm to standard DSA acquisitions. We realize, however, that because of the method of case selection, the possibility of selection bias impacting our results cannot be eliminated. Larger studies with strictly defined criteria for case selection are required to substantiate our observations. We believe, however, that our results are meaningful and that they provide a foundation on which to build a more definitive study.

Currently available flat panel detector DSA systems provide the means to acquire very high temporal and spatial resolution 2D images of the vasculature, bones, and soft tissues. The ability to derive physiologic information with this equipment is limited, largely as a result of the poor temporal resolution associated with the $\mathrm{C}$-arm movement, which is used to acquire 3D-DSA images. Application of color-coding algorithms to $2 \mathrm{D}$ acquisitions may offer 1 way of partially overcoming this restriction; it also provides a means to display (or visualize) an entire $2 \mathrm{D}$ sequence as a single composite image.

Very early in the development of DSA, the advantages inherent in a digital instead of an analog format for extraction and display of a large variety of parameters from image sequences became apparent. These parameters could be used as surrogates for some physiologic functions (eg, volume flow) ${ }^{3-5}$ While our study did not address the ability to assess 

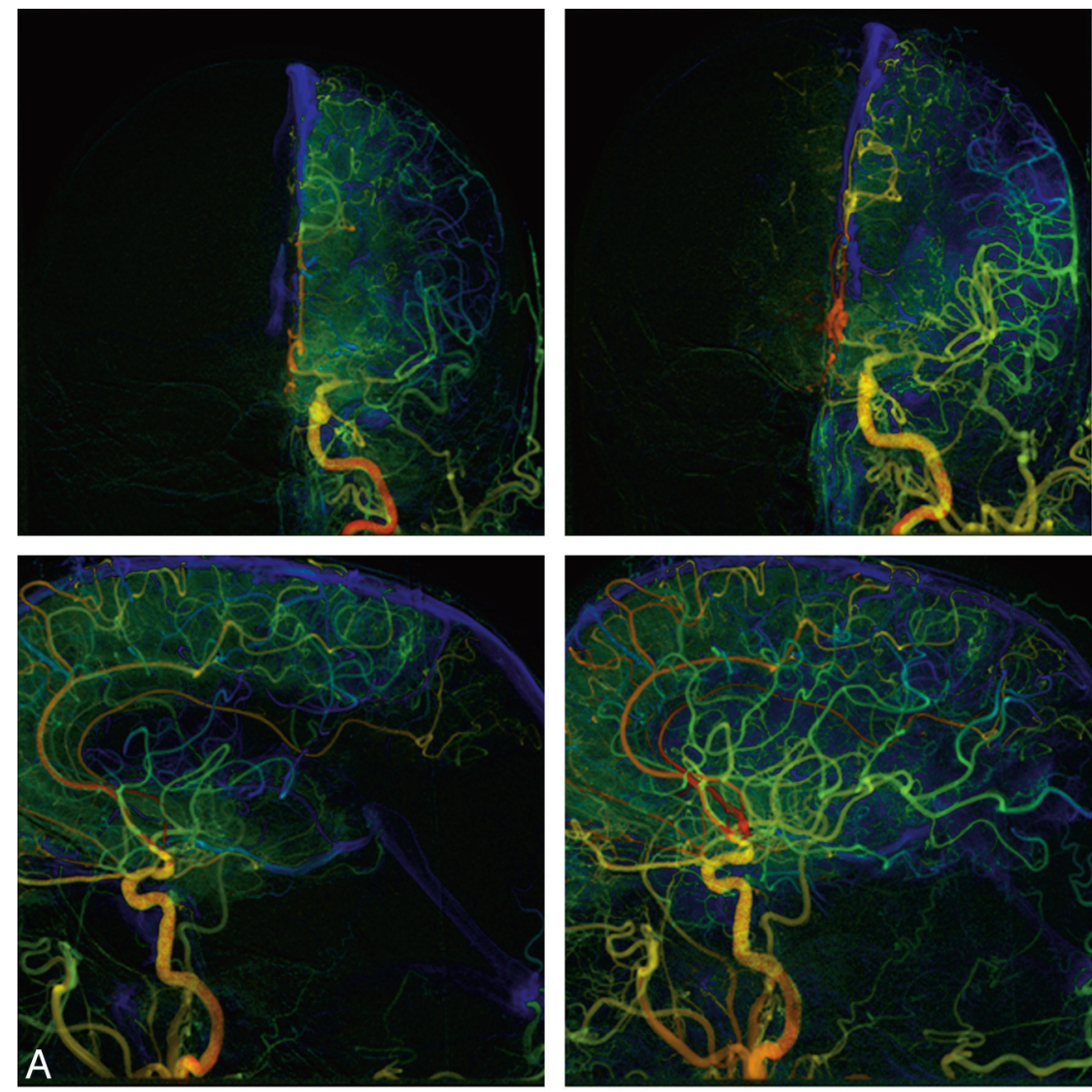

$1.3 \mathrm{~s}$

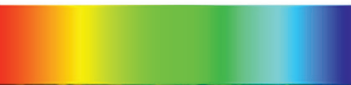

$6.7 \mathrm{~s}$
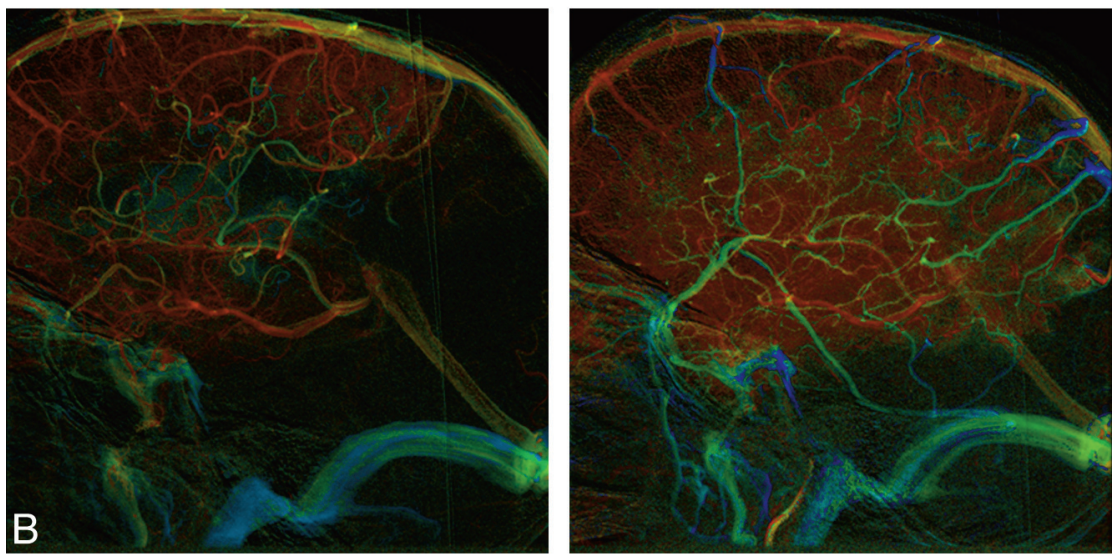

$6.8 \mathrm{~s}$

$11.5 \mathrm{~s}$

Fig 2. $A, A$ P and lateral color-coded images obtained before (left column) and after (right column) angioplasty and stent placement of a left middle cerebral artery stenosis. The posttreatment images clearly show faster arterial filling with greater opacification of the middle cerebral artery distribution. $B$, Color-coded images of lateral projections of the left internal carotid angiogram obtained before (left image) and after (right image) angioplasty and stent placement. Notice the greater extent of parenchyma opacification and more normal filling on the posttreatment image.

physiologic parameters directly, it did indirectly explore the use of the color-coding to measure some physiologic factors. For example, some of our test cases were from subjects who had been treated with angioplasty and/or stent placement for an intracranial stenosis or with angioplasty or drug infusion for postsubarachnoid-hemorrhage-induced vasospasm. In these instances, both pre- and posttreatment examinations were available for review. Here, the observers thought that the color-coded composite image displayed by using a TTP parameter provided better documentation of changes in flow of the contrast medium to and then through the parenchyma and, thus indirectly, of tissue perfusion than did the standard DSA sequences.

Extracting and displaying other parameters, such as time of 

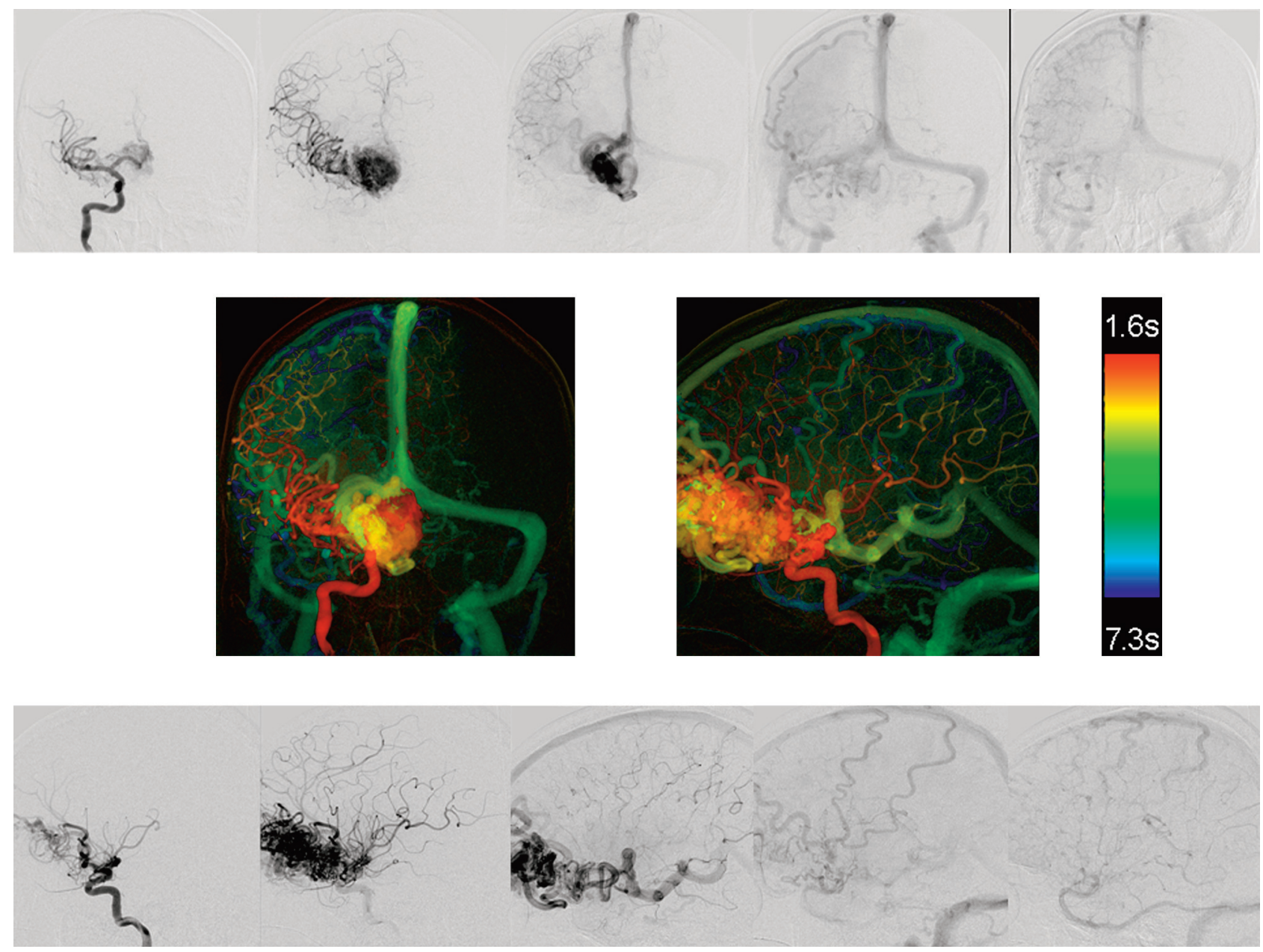

Fig 3. AP and lateral DSA images from a right internal carotid angiogram from a patient with a large frontal AVM (top and bottom rows). AP and lateral color-coded images from these acquisitions (middle row). The complex circulation of this AVM is clearly depicted on the color-coded composite images.

Table 2: Interobserver variability for each of the questions posed to the observers

\begin{tabular}{lc}
\hline Variable & Mean No. in Agreement \\
\hline Correct diagnosis, DSA & $5.27 \pm 0.19=87.82 \pm 3.14 \%$ \\
Correct diagnosis, DSA and & $5.23 \pm 0.2=87.18 \pm 3.37 \%$ \\
$\quad$ color-coded & \\
Color-coded impact on diagnosis & $4.19 \pm 0.17=69.87 \pm 2.78 \%$ \\
Color-coded impact on treatment & $4.62 \pm 0.17=76.92 \pm 2.79 \%$ \\
Color-coded impact on evaluation & $4.31 \pm 0.21=71.79 \pm 3.55 \%$ \\
$\quad$ of treatment success & \\
\hline
\end{tabular}

maximum increase or time of half wash-in, might provide even better indirect measures of tissue perfusion. While the color-coded composite image contains no added information to that present in the standard DSA sequence, it is impossible for a human observer to visually detect and analyze, on any DSA sequence or on any single DSA image, parameters such as TTP or time of maximum increase concentration, which may be important surrogates for physiologic events. ${ }^{3}$ Although it does not provide an absolute value of blood flow, TTP has been shown to be a useful parameter in estimating blood flow (eg, for indicating areas of the brain where blood flow is impaired and perfusion is abnormal). ${ }^{8}$ We believe that when used in conjunction with color-coded 2D-DSA, it, as well as other parameters such as time of maximum increase concen- tration, will prove useful in estimating blood flow and tissue perfusion, defining lesion size and location, and assessing changes after therapeutic interventions. Further work is ongoing to understand better the relationships between these parameters and the classic and more quantitative measurement of cerebral blood flow.

In some conditions such as AVMs, AVFs, and vascular tumors, the flow pattern is so complex and disturbed that multiple DSA acquisitions are required to fully document the anatomy and vascular filling pattern of the abnormality. As recently demonstrated by Cover et al, ${ }^{6}$ color-coding of a DSA acquisition seems to add value in allowing one to define the nidus of an AVM. A composite image of a complex AVM also allows visualization not only of the nidus of an AVM but also the simultaneous visualization of both normal and abnormal veins. Our observers thought that this capability provided a better means for treatment planning (both traditional operative removal and endovascular occlusion) than did the routine DSA sequences.

The color-intensity-projection algorithm described by Cover et $\mathrm{al}^{9}$ was proposed to evaluate object movement with the contrast arrival time and maximal enhancement being color-coded on a single composite image. In applying this to the AVMs, one determines the contrast medium arrival time indirectly by noting the difference between the mean intensity 
value and the minimal intensity value of a time-concentration curve. Because this approach uses only intensity information to determine contrast arrival time, it depends on an equally distributed (temporally) sample; this is not always the case because variable frame rate acquisitions are often used in angiography. Also, it seems likely that acquisitions obtained at high frame rates will provide some insight into flow within aneurysms. We are currently undertaking studies to determine just what frame rate is required to exploit this use of the algorithm as well as how the information obtained can be used to give insight into aneurysm flow characteristics. Application of the color-coded algorithm is rapid so that a composite image is available immediately after transfer of a DSA sequence to a dedicated workstation. The image is "free" in the sense that it requires no additional radiation exposure and no additional injection of contrast medium.

\section{Conclusions}

At no additional cost in $\mathrm{x}$-ray dose or contrast medium, color coding of DSA acquisitions enhanced the conspicuity of findings. It was particularly useful in situations in which there were complex flow patterns and in the evaluation of pre- and posttreatment acquisitions. Its full potential remains to be defined.

\section{References}

1. Cole BL, Maddocks JD, Sharpe K. Visual search and the conspicuity of coloured targets for colour vision normal and colour vision deficient observers. Clin Exp Optom 2004;87:294-304

2. D’Zmura M. Color in visual search. Vision Res 1991;31:951-66

3. Heintzen PH, Bürsch HJ, Hahne HJ, et al. Assessment of cardiovascular function by digital angiocardiography. J Am Coll Cardiol 1985:5:150S-57S

4. Cusma JT, Toggart EJ, Folts JD, et al. Digital subtraction angiograpic imaging of coronary flow reserve. Circulation 1987;75:461-72

5. Bürsch HJ. Funktionsangiokardiographie. Kardiologie 1989;78(suppl 7): $181-86$

6. Cover KS, Lagerwaard FJ, van den Berg R, et al. Color intensity projection of digitally subtracted angiography for the visualization of brain arteriovenous malformations. Neurosurgery 2007;60:511-15

7. Ahmed AS, Deuerling-Zeng, Strother CM, et al. Impact of intra-arterial injection parameters on arterial, capillary, and venous time concentration curves in a canine model. AJNR Am J Neuroradiol 2009;30:1337-41

8. Reichenbach JR, Röther J, Jonetz-Mentzel L, et. al. Acute stroke evaluated by time-to-peak mapping during initial and early follow-up perfusion CT studies. AJNR Am J Neuroradiol 1999;20:1842-50

9. Cover KS, Lagerwaard FJ, Senan S. Color intensity projections: a rapid approach for evaluating four-dimensional CT scans in treatment planning. Int $J$ Radiat Oncol Biol Phys 2006;64:954-61 